\title{
PAPR Reduction Method based on In-phase/Quadrature Data Symbol Components in MIMO-OFDM Systems
}

\author{
Mohammed I. Al-Rayif
}

\begin{abstract}
To overcome unpredictable spikes in the peak-toaverage power ratio (PAPR) in the presence of an orthogonal frequency-division multiplexing (OFDM) for multi-input-multioutput (MIMO) systems, implementation of a new SLM scheme is presented in this paper, which is extended from our previous study of IQ-SLM in SISO-OFDM system. In each transmit antenna, both real and imaginary parts of the base-band data symbol were modified independently using a corresponding phase element within a commonly generated phase vector, instead of modifying the complex data symbol as a single component. After applying an inverse fast Fourier transform (IFFT) for the real, imaginary, and original base-band vectors, the minimum PAPR component was observed. Therefore, the phase vector that introduced the minimal PAPR was considered to convert the original data block for transmission. This technique is called the In-phase/Quadrature-SLM (IQ-SLM) scheme. In this proposal, only $U$ phase vectors were generated to treat all $N_{t}$ data blocks, simultaneously, unlike the conventional MIMO-SLM techniques which generate $U N_{t}$ candidate phase blocks. The thing which, in turn, can be considered as a further computational complexity reduction, specifically in data-phase conversion stages. As a result, in terms of the complementary cumulative distribution function of PAPR performance(CCDF-PAPR), the proposal achieved a greater decibel reduction than conventional SLM methods such as dSLM, oSLM, and SSLM, at different subcarrier lengths $N$, candidate phase vectors $U$, transmit antennas $N_{t}$. Also, it shows a comparable BER performances over the dSLM scheme referencing to the theoretical curves, in the case where $N_{t} \leq N_{r}$ for both zero-forcing (ZF) and $\mathrm{ZF}$ with vertical Bell laboratories layered space-time (V-BLAST) detector.
\end{abstract}

Keywords-Peak-to-average power ratio (PAPR), ZF-VBLAST, selective mapping (SLM), I/Q-SLM, complementary cumulative distribution function (CCDF), MIMO-OFDM.

\section{INTRODUCTION}

A $\mathrm{HIGH}$ peak-to-average-power-ratio (PAPR) reduction in transmitter is essential in preventing the orthogonal frequency-division multiplexing (OFDM) signals from being clipped by non-linear high-power amplifiers (HPA). Increasing the linearity range of the power amplifier is optional solution; however, a shorter battery life and higher cost will be assumed

Manuscript received July 22, 2021; revised October 14, 2021. Date of publication November 15, 2021. Date of current version November 15, 2021. The associate editor prof. Gordan Šišul has been coordinating the review of this manuscript and approved it for publication.

Mohammed I. Al-Rayif is with the King Saud University College of Engineering, Saudi Arabia (e-mail: malrayif@ksu.edu.sa).

Digital Object Identifier (DOI): 10.24138/jcomss-2021-0123
[2], [3]. Thus, a PAPR reduction technique is demanded as another option to cope with this phenomenon. Hence, numerous proposals for PAPR reduction have been developed, for both single-input-single-output (SISO) and multi-inputmulti-output (MIMO) systems, including tone reservation [4], clipping and filtering [5] and [12], block-coding [6] and [11], and probabilistic methods [2], [7], [8]. All of these attractive methods help reduce PAPR but at the expense of system performance, efficiency, or computational complexity. Among these techniques, selected mapping (SLM) is the most commonly applied being free of distortion, hence it has been considered in this paper.

For SLM PAPR reduction in MIMO and massive-MIMO systems, a great number of proposals has been put forwarded. For instant, in the last few years, attractive schemes that deal with PAPR reduction via different mechanisms have been described [21]-[24]. Adaptive tone reservation scheme for PAPR reduction in large scale Multi-User MIMO-OFDM (MU MIMO-OFDM) systems was investigated by Chunxing Ni. et al. [21]. They found that only a segment of the signals has substantial power among all transmit antennas. An adaptive tone reservation (ATR) scheme was proposed to iteratively perform the TR scheme on the antenna with the maximum PAPR. Moreover, Bao et al. [22] represented a perturbation-assisted scheme and developed it to reduce the PAPRs of the transmitted signals by exploiting the redundant degrees-of-freedom inherent in the large-scale antenna array. In consequence, two efficient algorithms, referred to as PROXINF-ADMM1 and PROXINF-ADMM2, have been developed. Further, a letter by Long Chen et al. [23] proposed a two-step peak clipping (TPC) scheme to specifically decrease the PAPRs of the DM signals and presents a highly efficient DM transmitter with digital predistortion (DPD). However, these schemes suffer from the BER increment due to signal clipping, in addition to the fact that all these attractive proposals cannot be classified under the umbrella of distortionless (probabilistic) schemes. Moreover, by looking at a recent investigation method reported by Byung Moo Lee [24], attractive results via an SLM-based PAPR reduction scheme that can be applied to massive MIMOOFDM antenna systems based on antenna grouping was illustrated. Lee's basic idea was to divide the entire antenna system into several groups and apply a different set of phase 
sequences to each group. Then, various adjustable parameters for the performance complexity trade-off can be obtained. He called this method grouping-based concurrent SLM (gbcSLM). Further, he assigned more phase sequences for those antennas that had higher PAPR signals to improve PAPR performance more efficiently by sorting the antennas based on the PAPR of the OFDM signal of each antenna, hence further modified gbcSLMs were generated and were referred to as group-based different phase sequence concurrent SLMs (gbdpcSLM). This technique, however, emanated from the concurrent/simplified SLM (sSLM) [20], which will be considered in this paper for comparison purposes. While the obtained results in Lee were performed when applying a massive-MIMO system, the improvement was, somehow limited, while the number of candidate phase vectors $V$ was very high compared to the sSLM scheme, which introduced more complexity. Most earlier techniques, which are referred to as ordinary-SLM and simplified/concurrent-SLM (oSLM and sSLM/cSLM), were provided by Baek and Lee, respectively [19], [20]. Briefly, the oSLM scheme individually applies different $U$-SLM vectors to each of the $N_{t}$ parallel OFDM frames, where $N_{t}$ is the maximum number of transmit antennas, and the best phase modification for each $U$ group is individually selected, whereas, applying the same $U$-SLM vectors for all $N_{t}$-OFDM frames, simultaneously, is called sSLM (or cSLM). From this point on, the most famous directed-SLM (dSLM) scheme has been represented by Fischer and Siegle [18], in the dSLM scheme, the budget of $N_{t} U$-SLM vectors are applied to improve the currently highest PAPR over the antennas, only where needed. According to the investigated dSLM PAPR performance, the entire budget was specifically implemented such that the same complexity of $U N_{t}$ IDFTs as that of oSLM and sSLM was required. However, for dSLM, instead of applying only $U$ phase vectors per a transmit antenna, $U N_{t}$ phase vectors were generated and implemented which, in return, improved the performance better than the other methods, oSLM and sSLM. These techniques will be included in Section III, for comparisons purposes.

Based on the above survey and to the best of our knowledge, two studies depending on complex data decomposition have been reported regarding a PAPR reduction. The first is a technique developed in part by Sudha and Kumar [13], who described a low-complexity PAPR reduction in an SLMOFDM system using a time-domain sequence separation. Prior to the IDFT process, this method introduced a computational complexity in the same manner as a conventional SLM, where $U$ IDFTs copies are processed. Moreover, after the IDFT process, the real/imaginary and even/odd components were decomposed for a PAPR reduction, which likely introduced extra complexity. Next, GAO et al. [14] discussed a method for use in a MIMO-OFDM system, where the complex data components were decomposed to implement $V$ SLM vectors per component. However, this study reported a large value of the candidate vectors to be implemented at the OFDM, i.e. $V^{2}$, with only a small CCDF-PAPR reduction gained compared to conventional SLM-MIMO techniques.

This paper is extended based on the concept of IQ-SLM technique which was applied to the transmission side only of the SISO-OFDM system and proposed by Al-Rayif [1]. The idea of this proposal has been modified to be implemented in the transmitter/receiver MIMO-OFDM system. That is, for each transmit antenna, the real and imaginary parts of each transmitted base-band symbol are separated and converted into modified phases by the corresponding candidate phase element, which is generated randomly, as demonstrated in the following section. In addition, for selecting the best PAPR, this proposed method was expanded to consider the original PAPR instead of comparing only the modified PAPRs of the inverse discrete Fourier transform (IDFT) copies. This proposal shows an extreme comparable result in terms of CCDF-PAPR and BER performances, at the same computational complexity as the conventional MIMO PAPR-reduction schemes like oSLM, sSLM, and dSLM schemes. It is worth mentioning that the source of such a computational complexity is mainly from the IDFT operation, although the process of the PAPR reduction method itself can introduce an additional complexity under certain scenarios. Therefore, the comparisons in this proposal consider the fundamental complexity of the represented IDFT, with a PAPR reduction method, as a reference. The main contributions of this proposal are as follows:

- A comparable PAPR reduction can be obtained when considering the original PAPR at the stage of counting for the lowest PAPR.

- By applying only $U$ candidate phase vectors for all $N_{t}$ IDFT data blocks, the achieved PAPR reduction is still remarkable. Moreover, a significantly low number of bits of side information (SI) is needed, in case the designed system requires the transmission of SI, in contrast with dSLM and oSLM schemes.

- Regarding the computational complexity of the IDFT system, the complexity of the proposed approach and the oSLM, sSLM, and dSLM schemes can be considered equal ( $U N_{t}$-IDFTs), owing to the fact that the proposed real and imaginary parts are real quantities when each component is IDFT-processed separately.

- For testing the system performances, in terms of bit error rate (BER), both schemes dSLM and the proposed IQ-SLM were recovered without transmitting any side information. Keeping in mind that both schemes of IQ-SLM [1] and dSLM [18] were represented at only the transmission stage, hence, in this paper, its receivers have been designed as appropriate for the MIMO-OFDM system by coupling them with other schemes as follows. The approach of [2] and [16] was implemented with the proposed IQ-SLM to recover the original data at the receiver, whereas the recovery method of [17], partial orthogonal SLM (POSLM), was implemented with dSLM for conversion phases purposes. Based on that, BER performances have been investigated by the sub-optimal ZF and ZF-VBLAST detection. Accordingly, the proposed IQ-SLM showed improvement and superior performance to the other technique at $N_{t} \leq N_{r}$.

The remainder of this paper is organized as follows: The proposed system model is provided in Section II, followed by the simulation results and discussion in Section III. Finally, in Section IV, some concluding remarks are provided. 


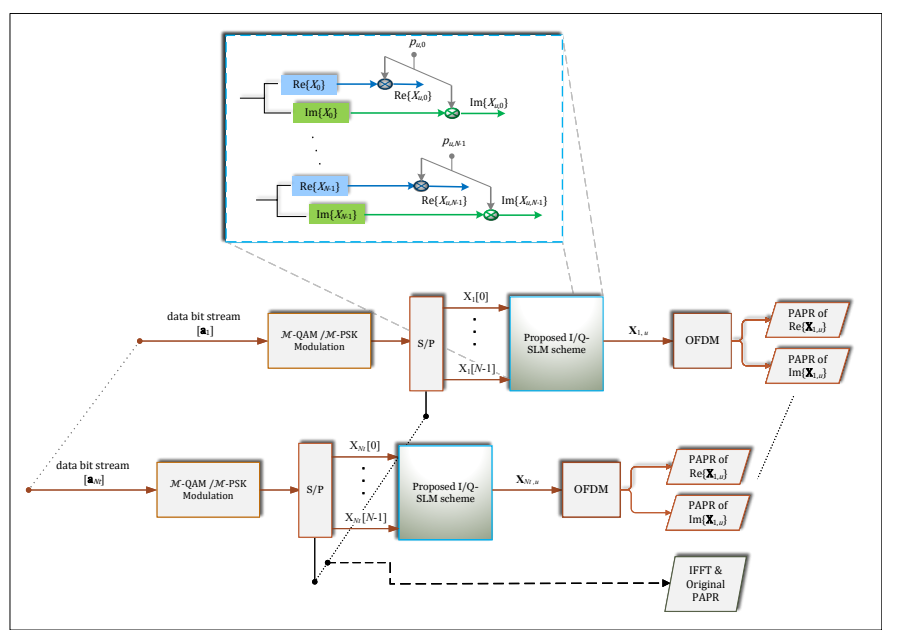

Fig. 1. Block diagram of the proposed IQ-SLM at the MIMO transmitter side

\section{Proposed System Model}

Based on the block diagram depicted in Fig. 1, assume $N_{t}$ transmit antennas, over which mutually independent data streams are communicated with $N_{r}$ receive antennas. Also, consider a discrete time OFDM system model, employing an inverse discrete Fourier transform (IDFT) of length $N$ active carriers, and in each of the $N_{t}$ parallel OFDM transmitters, binary data is mapped to complex-valued data symbols $X_{k}$ taken from an $M$-ary QAM constellation with mean $E\left\{\left|X_{k}\right|\right\}$ and variance $E\left\{\left|X_{k}\right|^{2}\right\}=\sigma_{X}^{2}$, for $0 \leq k \leq N-1$. For $i$-th transmit antenna, the frequency domain OFDM frames denoted by $\mathbf{X}_{i}=\left[X_{i, 0}, X_{i, 1}, \ldots, X_{i, k}, \ldots, X_{i, N-1}\right]^{T}$ are transformed into the time domain OFDM frames $\mathbf{x}_{i}=$ $\left[x_{i, 0}, x_{i, 1}, \ldots, x_{i, n}, \ldots, x_{i, N-1}\right]^{T}$, for $0 \leq n \leq N-1$, where $[\cdot]^{T}$ is the transpose operation. The correspondence is written in short as $\mathbf{x}_{i}=\operatorname{IDFT}\left\{\mathbf{X}_{i}\right\}$. As for the concept of the proposed IQ-SLM scheme, suppose a single typical OFDM, so that the " $i$-th" index of the transmitter can be omitted for the moment. And then, each frequency-domain complex symbol is decomposed into its real $\Re\left\{X_{k}\right\}$ and imaginary $\Im\left\{X_{k}\right\}$ parts for all $k$, simultaneously. Thereafter, each $k$-th component is multiplied with the corresponding element of the random candidate phase element $p_{k}^{u} \forall u \in\{1, \ldots, U\}$, where $p_{k}^{u} \in\{-1,1\}$, and $p_{k}^{0}=[1]$, for all $k$ with $0 \leq k \leq N-1$, to generate the original phases stream. For OFDM operation, an IDFT is applied for each modified component to obtain the $n$-th subcarrier of the real, imaginary, and original sequences, that is $x_{n, R}^{u}=\operatorname{IDFT}\left\{\Re\left\{X_{k}^{u}\right\}\right\}, x_{n, I}^{u}=\operatorname{IDFT}\left\{\Im\left\{X_{k}^{u}\right\}\right\}$, and $x_{n, \text { Org }}=$ IDFT $\left\{X_{k, \text { Org }}\right\}$, respectively, where $X_{k, \text { Org }}$ is the original data symbol without the effect of any $u$-th phase sequence and $n \in\{0,1, \ldots, N-1\}$. Hereafter, the peak-toaverage-power ratio (PAPR) is accounted for in each OFDM outcome signal to get a list of $\mathrm{PAPR}_{\text {Org }}, \mathrm{PAPR}_{u, R}$, and $\mathrm{PAPR}_{u, I}$ where, generally, the PAPR is expressed as follows:

$$
P A P R=\frac{\max _{n}\left[\left|x_{n}\right|^{2}\right]}{E\left[\left|x_{n}\right|^{2}\right]}
$$

By extending this concept to MIMO-OFDM system, for each transmit antenna, the same described processes are implemented. Consequently, the $u$-th OFDM symbol of $i$-th transmit antenna with the lowest PAPR, $\mathbf{x}_{i}^{u^{\prime}}$, is considered for transmission, i.e.,

$$
\mathbf{x}_{i}^{u^{\prime}}=\mathbf{x}_{i}^{u}\left\{\underset{0 \leq u \leq U-1}{\operatorname{argmin}}\left[P A P R_{i}^{O r g}, P A P R_{i}^{u, R}, P A P R_{i}^{u, I}\right]\right\},
$$

As for CCDF-PAPR counting, depending on the description of Equation (3) in [18], which expressed the CCDF-PAPR of the conventional SISO SLM as $\left(1-\left(1-e^{-\zeta_{t h}}\right)^{N}\right)^{U}$, and taking into consideration that in this proposal the $U$ are implemented separately to the real and imaginary parts, hence the joint probability can be formulated by $\left(1-\left(1-e^{-\zeta_{t h}}\right)^{N}\right)^{U^{2}}$, where $\zeta_{t h}$ is a certain threshold which is exceeded by the measured OFDM-PAPR. Since we count for the worst-case of this probability for all given $N_{t}$, the theoretical CCDF-PAPR in MIMO-OFDM system can be approximated to

$$
\operatorname{Pr}\left\{P A P R_{u^{\prime}}>\zeta_{t h}\right\}=\left(1-\left(1-e^{-\zeta_{t h}}\right)^{N}\right)^{N_{t} U^{2}} .
$$

Here, the maximum PAPR ${ }_{i}$ is considered for the worst case. Finally, the received $\left[N_{r} \times N\right]$ matrix $\mathbf{y}$ can be expressed as $\mathbf{y}=\mathbf{H} \mathbf{x}^{v}+\eta$ where $\mathbf{H} \sim \boldsymbol{N}_{c}\left(0, \boldsymbol{I}_{N_{r}}\right)$ is the $\left[N_{r} \times N_{t}\right]$ perfectly known matrix of Rayleigh fading channel which contains uncorrelated complex Gaussian fading gains, $\mathbf{x}^{v}$ is the $\left[N_{t} \times N\right]$ matrix of transmit symbols to which phases have been converted based on the phases of vector $v$ where $v \in\{0,1, \ldots, U\}$, and $\eta \sim \boldsymbol{N}_{c}\left(0, \sigma_{\eta}^{2} \boldsymbol{I}_{N_{r}}\right)$ is $\left[N_{r} \times N\right]$ matrix of i.i.d white complex Gaussian noise.

Thereafter, to reshape this received signal back to the original phase vector, the technique represented by [2], [16] was implemented with this proposal (IQ-SLM), whereas the scheme of [17] was implemented with dSLM and both detected by the sub-optimal zero-forcing (ZF) and ZF-VBLAST detectors without explicit side information. In brief, both methods introduced an extension factor $C>1$ at a specific elements of the candidate phase vector according to the concept of each method. From this point on, at the receiver side, the recovery model of [16] can estimate the desired phase vector $\mathbf{P}^{v}$ for each transmitted frame of the proposed IQ-SLM (in MIMO-OFDM system) via the following metric; $\alpha_{v}=\underset{0<u<U-1}{\arg \min }\left\{\left.\left|E\left[|\mathbf{Y}|^{2}\right]-\sigma_{\eta}^{2}-\Gamma\right| \mathbf{H}\right|^{2}\left(\mathbf{P}^{u}\right)^{2} \mid\right\}$, where $\Gamma$ denotes the average energy per transmitted complex symbol, $\mathbf{Y}=\operatorname{DFT}\{\mathbf{y}\}$, and $\mathbf{P}^{u}$ is the library of the generated phase vectors at the receiver. While the recovery model of [17] can be expressed as $\mathbf{P}^{v}=\underset{0<u<U-1}{\arg \max }\left\{\sum_{n=\beta+1}^{N-1}|Y[n]|^{2}(p[n])^{2}\right\}$ to estimate the received phase vector of dSLM scheme, noting that only the subblock of the candidate phases from element $\beta+1$ was generated orthogonally, and hence extending only the positive elements to the factor $C>1$. Whereas, in the proposed IQ-SLM, because all phase blocks were generated randomly, hence each subblock with length $l=\frac{N}{U}$ per a phase vector was extended to the same value of $C$ whether the element was positive or negative, as represented in [2]. Under these assumptions, the increase energy per transmitted 
block can be determined as $10 \log _{10}\left[\frac{N\left(C^{2}-1\right)}{U}+1\right](\mathrm{dB})$ for the proposed scenario, and $10 \log _{10}\left[\frac{\beta\left(1-C^{2}\right)+N\left(1+C^{2}\right)}{2 N}\right](\mathrm{dB})$ for the dSLM scenario.

\section{NumericAl Results AND Discussions}

In this section, characteristics of the proposed scheme are numerically investigated. This investigation is based on an I/Q decomposition, in terms of CCDF-PAPR reduction and bit error rate (BER) in MIMO-OFDM system. The numerical simulations were obtained at various parameters, including the OFDM block length $N$ (subcarriers/block/transmitter), transmit $N_{t}$ and receive $N_{r}$ antennas, the input-back-off (IBO) values, and the number of candidate phase vectors $U$. Last but not least, it is worth mentioning that the PAPR of discrete time samples were generated, which gives an estimate of the PAPR of continuous time signals when adding an oversampling factor $N_{o s}=4$ to the discrete OFDM frames. For simplicity, such a factor is omitted from the formulas of this paper.

\section{A. CCDF-PAPR Performance and Computational Complexity in MIMO-OFDM}

As mentioned above, this study considered the schemes dSLM, oSLM, and sSLM for comparison purposes where all depended on the SLM concept. It is also worth noting that the dSLM scheme showed superior performance over the sSLM and oSLM. Hence, based on the number of IDFTs (computational complexity), all methods, including the proposed IQ-SLM, were equivalent, i.e., $N_{t} U$-IDFTs plus the original $N_{t}$-IDFT. However, as for the complexity of the SLM conversion process, treatment with $U$ phase vectors (like the proposal and sSLM) performed at a lower complexity than $N_{t} U$ vectors (like oSLM and dSLM). Moreover, in terms of the number of bits side information (SI) required for recovery procedure, the proposed version and SSLM scheme were nearly the same, i.e., only $\left\lceil\log _{2}(U+1)\right\rceil$ and $\left\lceil\log _{2}(U)\right\rceil$, respectively. This was because all $N_{t}$ IDFTs were modulated with the same generated $U$ phase block, aside from the original data phase block, in the proposed version. Unlike the dSLM, which is operated with a budget of $N_{t} U$ phase blocks, $N_{t}\left\lceil\log _{2}\left(N_{t} U+1\right)\right\rceil$ bits SI were required, while in the oSLM version, $N_{t}\left\lceil\log _{2}(U)\right\rceil$ bits SI were required, to which each transmit antenna generated a different $U$ phase blocks. For example, if $N_{t}=4$ antennas, and $U=4$ phase blocks, then the proposed scheme needed about 3 bits SI, whereas about 16 bits SI were required in the dSLM version. In this paper, none of these procedures was necessary because the implemented system needed no SI for recovery of original data phases. According to the CCDF-PAPR characteristics, Figs. 2-4 display the CCDFPAPR for the proposed IQ-SLM, sSLM, oSLm, and dSLM schemes. In Fig.2, it is visible that the proposed IQ-SLM performed superbly compared with the dSLM for all numbers of transmit antennas $N_{t}$. Such was the case when fixing the number of transmit antennas and candidate phase blocks to $N_{t}=4$ and $U=4$, respectively, while varying the number of subcarriers/block to $N=64$ and 512 , as illustrated

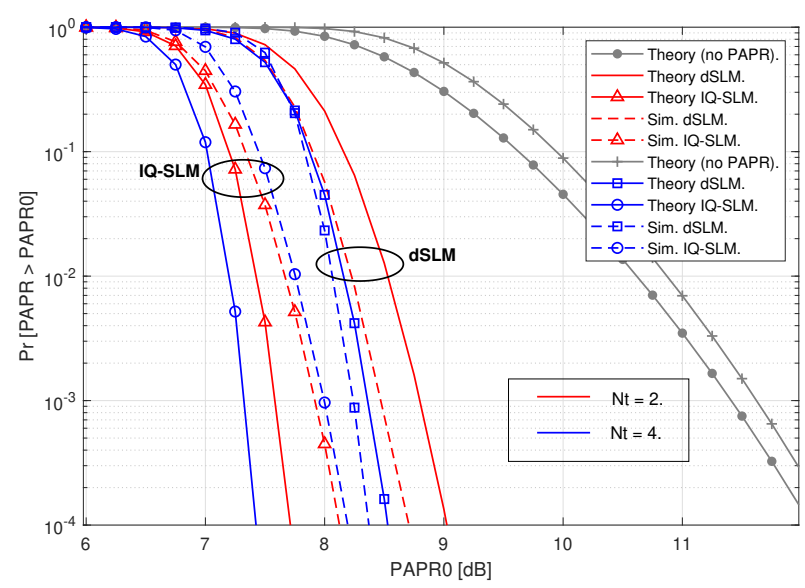

Fig. 2. PAPR reduction for multiple transmit antennas (MIMO) at different numbers of $N_{t} ; N=512$ subcarriers, $U=4$ phase vectors.

in Fig.3. It should be noted that the reduction gain of the proposed IQ-SLM was achieved by implementing only $U$ phase blocks to all transmit antennas, while in the dSLM, $U N_{t}$ phase blocks were implemented to reach the illustrated PAPR reductions. More specifically, in Fig.4, for $N=256$ subcarriers/block, $U=4$ phase blocks and $N_{t}=4$ transmit antennas, equivalent budget of phase blocks was generated, i.e., $U_{S S L M}=U_{o S L M}=U_{d S L M}=U_{I Q S L M}=16$ phase vectors implemented for all schemes to represent the favorable results. Once again, the proposed IQ-SLM performed better than all other techniques and was able to gain more than $5 d B$ from the original PAPR reduction, and about $1.25 \mathrm{~dB}$ from the dSLM scheme.

To conclude, two main reasons could be stand behind these behaviors of results. The first is from the fact that the norm value of the complex symbol is greater than that of a real or imaginary part separately. Therefore, the overall average power and the maximum peak are reduced in IQSLM, and in consequence, the PAPR ratio is reduced as well. The second possible reason is that when applying $U$ phase vectors randomly on each separated real and imaginary component, the similarity of each OFDM copy to the other can be reduced to the best level, particularly when having larger number of differences (choices) of data conversions. As a consequence, the chance to obtain a lower PAPR is increased as demonstrated in the simulation results. This is not what happens when completely non-random phase vectors (partly orthogonal phase vectors) $U$ are implemented.

\section{B. Bit Error Rate (BER) Performance}

This section studies the impact of the proposed IQ-SLM on the performance of the MIMO-OFDM system in terms of BER, in comparison to the dSLM scheme at the same inputs. Considering that, as mentioned in Section-II, the proposed IQ-SLM structure depends on $l$ extended elements, for recovery purpose, as represented in [2] and [16], while the dSLM system depends on the concept of POSLM recovery as discussed in [17] with $\beta \ll N$ and $(N-\beta)$ is the number 


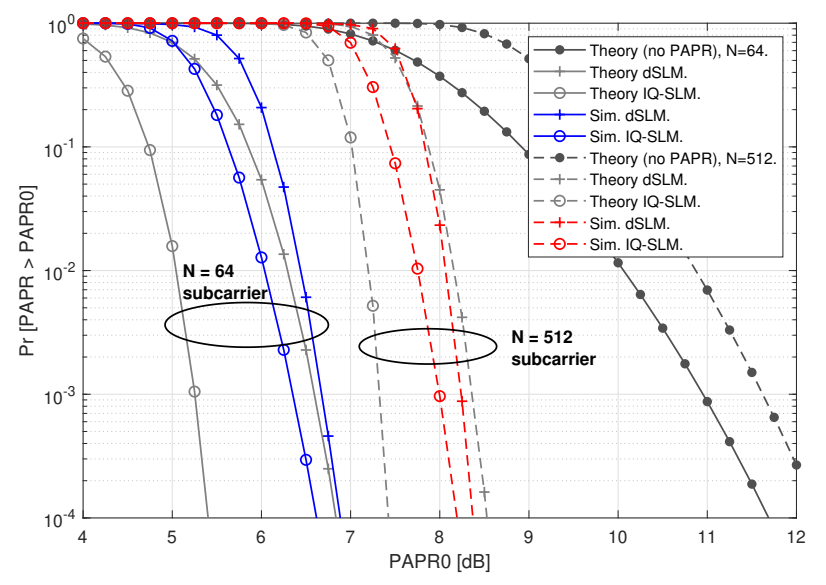

Fig. 3. PAPR reduction for multiple transmit antennas (MIMO) at different values of $N$-subcarriers; $N_{t}=4$ antennas, $U=4$ phase vectors.

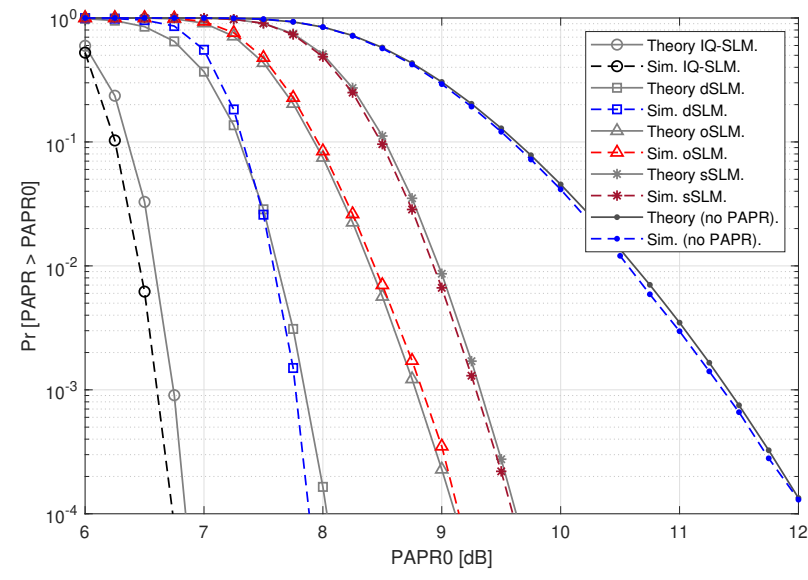

Fig. 4. PAPR reduction for multiple transmit antennas (MIMO) at $U_{O S L M}=$ $U_{S S L M}=U_{d S L M}=U_{I Q S L M}=16$ phase blocks, $N=256$ subcarriers, $N_{t}=4$ antennas.

of orthogonal elements in which only its positive elements are extended. From this point on, performances were obtained at different number of transmit $N_{t}$ and receive $N_{r}$ antennas, $N$ subcarriers/ frame/ transmit antenna, non-linear power amplifier input-back-off (IBO), extension factor $C$, and different length of parameter $l=\frac{N}{U}$. Accordingly, we can classify the obtained results into two groups: the first group for which the number of transmit and receive antennas are equal, $N_{t}=N_{r}$, and the results for which the number of transmit antennas are less than the receivers, $N_{r}>N_{t}$, as drawn in Figs. 5 and 6 , respectively. Looking at Fig. 5, it is possible to observe that with increasing the length of a transmitted OFDM block $N$ while fixing all other inputs, as illustrated in Fig.5a, Fig. 5b, and Fig. 5c, the overall performances are mended, however it is interesting to note that the performances of the proposed IQ-SLM is much superior to the dSLM performances, for all cases. Also, it can be demonstrated that the estimated signals of the proposal technique outperform the other when implementing the ZF-V-BLAST detector. Furthermore, Fig.
6 displays the BER performances at $N_{r}>N_{t}$ and the input-back-off is increased from IBO $=5 \mathrm{~dB}$ (Fig.6a) to IBO $=7 \mathrm{~dB}$ (Fig.6b). This figure shows how the increase in the number of receive antennas participates in improving both ZF and ZF-VBLAST performances, in comparison to the previous figure. On the other hand, from Fig. (6a), we can recognize that, despite the proposed IQ-SLM superiority, its performance diverges from the theoretical lines, which is due to the impact of IBO decreasing, even with high $E_{b} / N_{o}$. Whereas in Fig. (6b), the estimated IQ-SLM are very near to the theoretical lines, unlike the dSLM scheme which still suffers from the IBO effect. To end, the small differences between the theoretical lines and the estimated lines of the proposal can be caused by the extension factor, that is $C=1.5$. Accordingly, the reason behind this distinction of the proposed system IQ-SLM can be summarized in the following factors. The first is due to the structure of the generated POSLM phase sequence itself, which is designed to consist of two parts: the first is random (up to $\beta$ ) and the second is orthogonal (from $\beta+1$ to $N$ ), so that the latter is used to reconstruct the original phases at the receiver side. Thus, the shorter the length of the generated phase vector, the shorter the length of the orthogonal part, and thus the reconstruction process resulted in more errors in the side information, as illustrated in Fig.7. From this figure, it can be noticed that the dSLM scheme (via POSLM) needs higher value of $C$, i.e. extra energy, to achieve the same performance of the proposal (IQ-SLM), for all inputs of IBO $(\mathrm{dB})$ and SNR $(\mathrm{dB})$. For instance, at SIER $=10^{-3}$, the dSLM scheme requires extra extended value to $(\approx 0.4)$ to perform as the same as the proposed IQ-SLM, at both low and high $\mathrm{SNR}=10$ and $30 \mathrm{~dB}$. The second factor is the amount of increase energy per a frame when injecting the extension factor $C$ inside the generated phase vector, for recovery process. To clarify, assume the length of the generated phase sequence is $N=128$ elements for both schemes, IQ-SLM and POSLM. Hence, based on the POSLM design, only the +1 s inside the orthogonal part (say at $\beta=\frac{N}{2}=64$ ) is extended to $C$ (say $C=1.5$ ). However, in the proposed IQ-SLM, $l$ elements are extended to $C$. In other words, if $U=4$ candidate phase vectors, then $l=\frac{N}{U}=32$ elements/vector must be extended to $C=1.5$ either the element was +1 or -1 . Consequently, the overall increased energy for the IQ-SLM is greater than that of POSLM, therefore the recovery metric of the proposal operates better than the recovery metric of POSLM. Fig. 8 demonstrates a comparisons between the increase energy versus extension factor $C$ for both POSLM and IQ-SLM at different lengths of subvector $l, N=128$ elements/block and $\beta=64$. This figure shows that the POSLM technique requires lower energy than the proposed IQ-SLM scheme for all values of $N \geq l \geq 1$ which is a logical reason behind the improvement in the IQSLM BER performance, but at the expense of the energy consumed. Should the reader need further details about these schemes, it is recommended to see the proposal of [2], [16], and [17].

\section{CONCLUSION}

In this paper, I/Q-SLM PAPR reduction method based on In-phase and Quadrature data symbol components was 


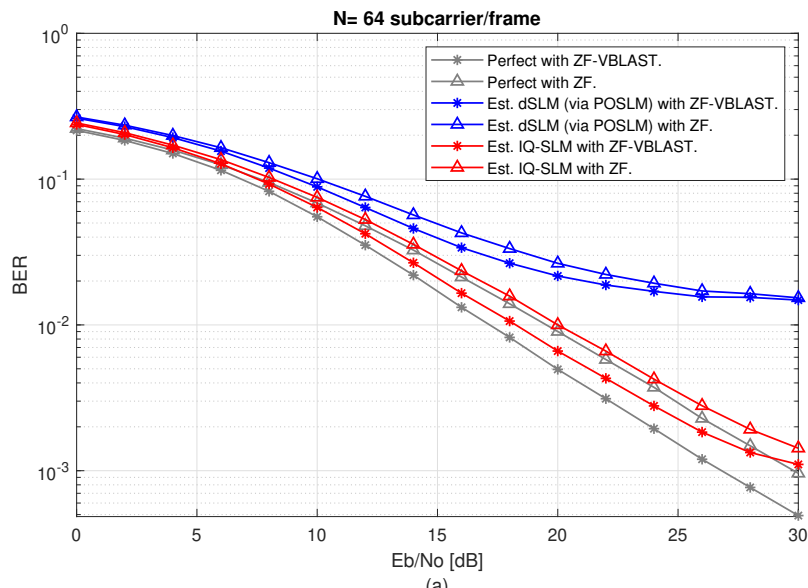

(a)

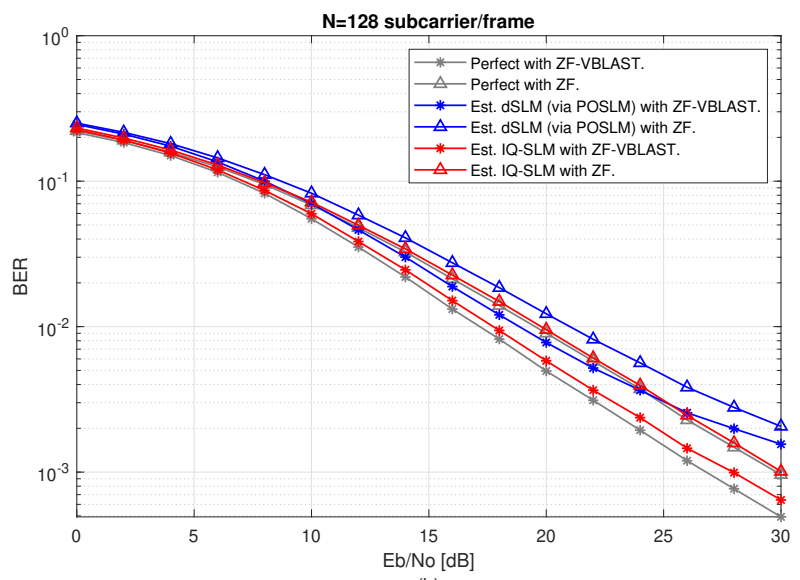

(b)

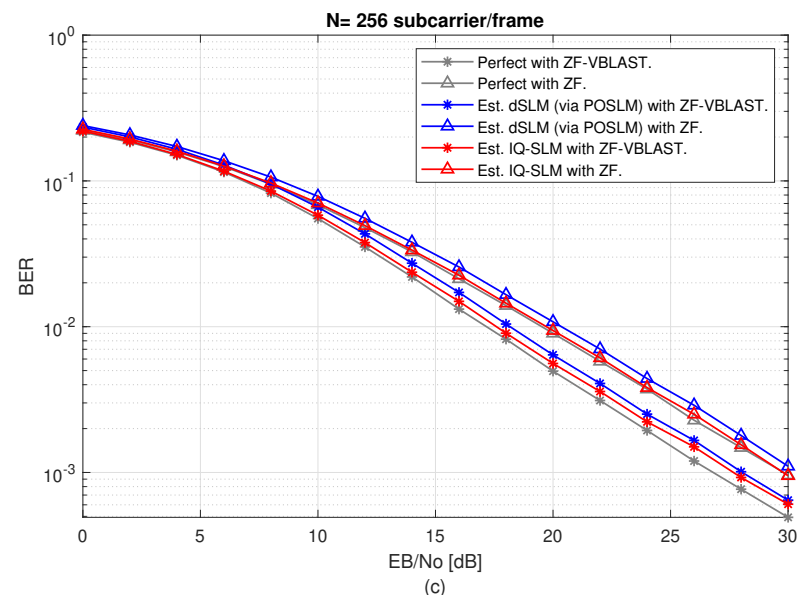

Fig. 5. I/Q-SLM and dSLM BER for 16-QAM MIMO-OFDM signals at different numbers of subcarriers/transmitter $(N=64,128$, and 256) for $N_{t}=N_{r}=2$ antennas, $U=4$ phase vectors, IBO $=7 \mathrm{~dB}$, and $C=1.5$.

represented for MIMO-OFDM system. A set of random phase blocks were generated, for all transmit antennas, to convert the original phases of data sequence after decomposing its real and imaginary parts separately. By considering the lowest obtained CCDF-PAPR reduction from either the real, imaginary, or original PAPRs, the lowest PAPR was introduced for each transmitter, simultaneously, then the maximum $\mathrm{PAPR}_{i}$ of the $i$-th antenna was selected for drawing the CCDF-PAPR characteristics. The performance of this technique was shown

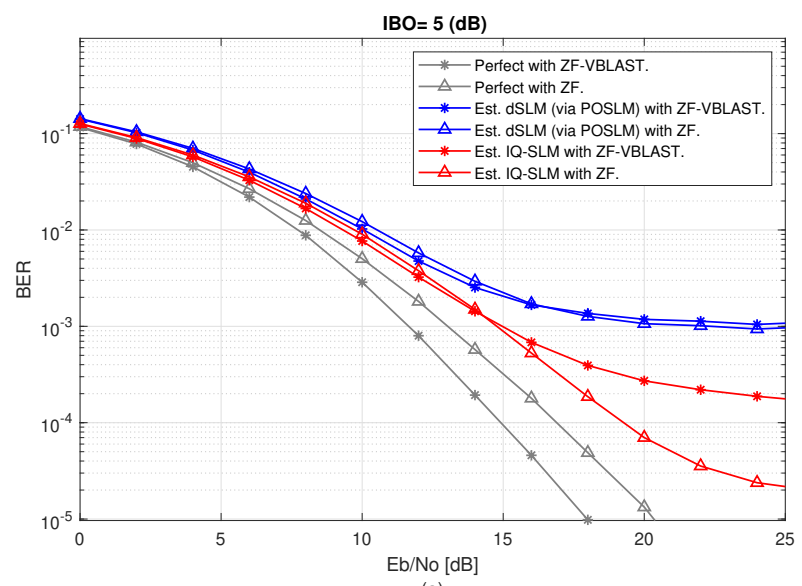

(a)

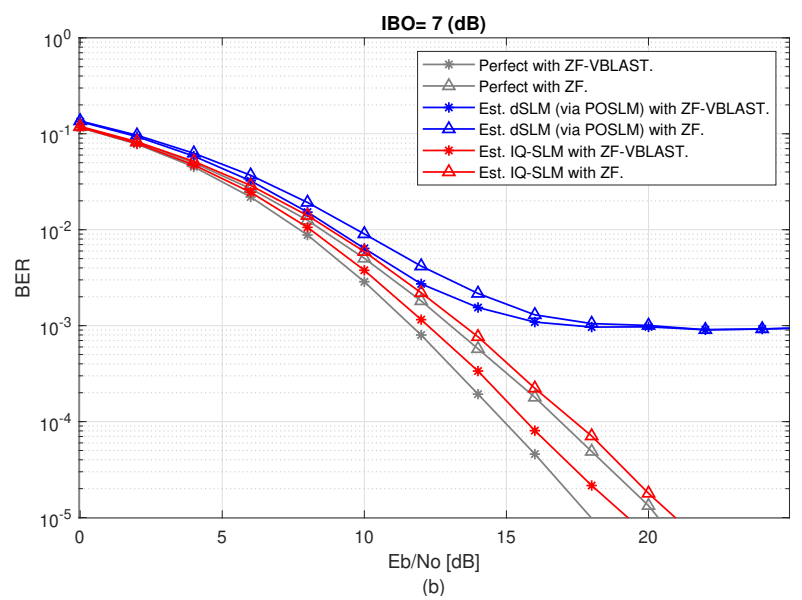

Fig. 6. I/Q-SLM and dSLM BER for 16-QAM MIMO-OFDM signals at different input-back-off for $N_{t}<N_{r}\left(N_{t}=2, N_{r}=4\right)$ antennas, $U=4$ phase vectors, $N=128$ subcarrier/transmitter, and $C=1.5$.

at different values of $N$ subcarriers, $U$ candidate phase blocks, $N_{t}$ transmit and $N_{r}$ receive antennas, and input-back-off (dB). The results of the proposed IQ-SLM showed impressive and superior achievement in terms of PAPR and BER performances. The PAPR was reduced by approximately 1,2 , and $2.5 \mathrm{~dB}$ from the dSLM, oSLM, and sSLM curves, respectively, at $N=256$ subcarriers and $P_{r}\left[P A P R>P A P R_{0}\right]=10^{-4}$, specifically, at $U_{I Q S L M}=U_{d S L M}=U_{o S L M}=U_{S S L M}=16$ phase vectors. In addition, this proposal represents a satisfactory BER performances when $N_{t} \leq N_{r}$ in comparison with the performances of dSLM scheme, when implementing both $\mathrm{ZF}$ and ZF-VBLAST detectors. Nevertheless, this is at expense of energy consumed per a transmitted phase vector, which can be considered as a future study.

\section{ACKNOWLEDGMENT}

The author would like to thank the stuff of the Researchers Support Services Unit (RSSU) in King Saud University for their help and support in making this work possible.

\section{REFERENCES}

[1] M. I. Al-Rayif, "Optimal SLM PAPR Reduction based on I/Q-Complex Data Symbol Components," presented at meeting (2nd IEEE Middle East and North Africa COMMunications Conference (MENACOMM)), Manama, 2019, pp. 1-4, doi: 10.1109/MENACOMM46666.2019.8988519. 


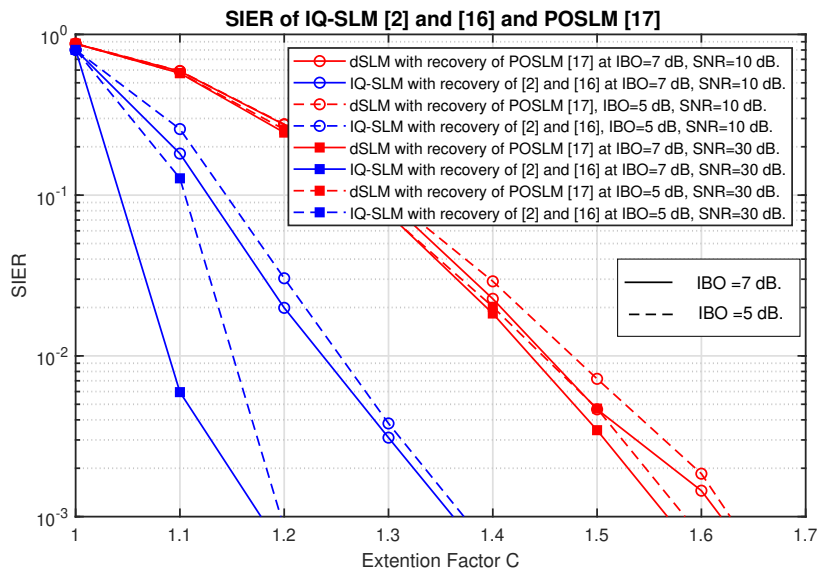

Fig. 7. Side information error rate (SIER) versus different levels of extension factor $C$ at $U=4$ phase blocks, $N=128$ subcarriers, $N_{t}=2$ antennas, and $\beta=64$ orthognal elements/frame with $\mathrm{SNR}=10$ and $30 \mathrm{~dB}$.

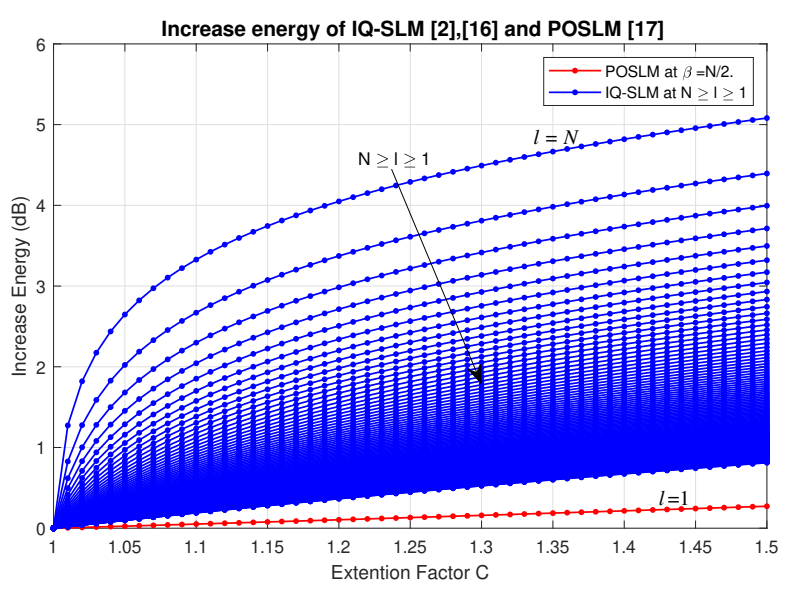

Fig. 8. Comparisons of increase energy $(\mathrm{dB})$ per a frame versus different levels of extension factor $C$ at $U=4$ phase blocks, $N=128$ subcarriers, $N_{t}=2$ antennas, and $\beta=64$ orthognal elements/frame.

[2] M. I. Al-Rayif, H. Seleem, A. Ragheb and S. Alshebeili, "A Novel Iterative-SLM Algorithm for PAPR Reduction in 5G Mobile Fronthaul Architecture", IEEE Photonics Journal, vol. 11, no. 1, pp. 1-12, 2019, Art no. 7201112. doi: 10.1109/JPHOT.2019.2894986

[3] S. Wang, K. Lee and C. Li, "A Low-Complexity Architecture for PAPR Reduction in OFDM Systems With Near-Optimal Performance", in IEEE Transactions on Vehicular Technology, vol. 65, no. 1, pp. 169-179, 2016. doi: 10.1109/TVT.2015.2395818

[4] Y. Wang, R. Zhang, J. Li and F. Shu, "PAPR Reduction Based on Parallel Tabu Search for Tone Reservation in OFDM Systems," in IEEE Wireless Communications Letters, vol. 8, no. 2, pp. 576-579, 2019. doi: 10.1109/LWC.2018.2880432

[5] K. Anoh, C. Tanriover, B. Adebisi and M. Hammoudeh, "A New Approach to Iterative Clipping and Filtering PAPR Reduction Scheme for OFDM Systems," in IEEE Access, vol. 6, pp. 17533-17544, 2018. doi: 10.1109/ACCESS.2017.2751620

[6] R. Yoshizawa and H. Ochiai, "Energy Efficiency Improvement of Coded OFDM Systems Based on PAPR Reduction,” in IEEE Systems Journal, vol. 11, no. 2, pp. 717-728, 2017. doi: 10.1109/JSYST.2015.2469783

[7] M. I. Al-Rayif , H. Seleem, A. Ragheb, and S. Alshebeili, "Experimental Demonstration for PAPR Reduction in OFDM System using PartialOSLM Technique," IEEE Journal of Circuits, Systems, and Computers, vol.27, no. 7, pp. 1-12, 2017.

[8] J. Ji, G. Ren and H. Zhang, "A Semi-Blind SLM Scheme for PAPR
Reduction in OFDM Systems With Low-Complexity Transceiver," in IEEE Transactions on Vehicular Technology, vol. 64, no. 6, pp. 26982703, 2015. doi: 10.1109/TVT.2014.2345262

[9] A. M. Rateb and M. Labana, "An Optimal Low Complexity PAPR Reduction Technique for Next Generation OFDM Systems," in IEEE Access, vol. 7, pp. 16406-16420, 2019. doi: 10.1109/ACCESS.2019.2895415

[10] W. Hu, "PAPR Reduction in DCO-OFDM Visible Light Communication Systems Using Optimized Odd and Even Sequences Combination," in IEEE Photonics Journal, vol. 11, no. 1, pp. 1-15, 2019, Art no. 7901115. doi: 10.1109/JPHOT.2019.2892871

[11] M. Kim, W. Lee and D. Cho, "A Novel PAPR Reduction Scheme for OFDM System Based on Deep Learning," in IEEE Communications Letters, vol. 22, no. 3, pp. 510-513, 2018. doi: 10.1109/LCOMM.2017.2787646

[12] M. A. Khan and R. K. Rao, "Low-Complexity PAPR Reduction Technique for OFDM Systems Using Biased Subcarriers," in Canadian Journal of Electrical and Computer Engineering, vol. 39, no. 1, pp. 19-25, 2016. doi: 10.1109/CJECE.2015.2492919

[13] V. Sudha, and D.S. Kumar, "Low complexity PAPR reduction in SLM-OFDM system using time domain sequence separation," Alexandria Engineering Journal, vol. 57, pp.3111-3115, 2018. doi:10.1016/j.aej.2017.11.006.

[14] J. Gao, J. Wang, and Z. Xie, "Peak to Average Power Ratio Reduction for MIMO-OFDM Systems with Decomposed Selected Mapping," International Journal of Information and Systems Sciences, vol. 3, no. 3-4, pp. 572-580, 2009.

[15] R. van Nee and A. de Wild, "Reducing the Peak-to-Average Power Ratio of OFDM," VTC '98. 48th IEEE Vehicular Technology Conference. Pathway to Global Wireless Revolution (Cat. No.98CH36151), Ottawa, Ont., 1998, pp. 2072-2076 vol.3. doi: 10.1109/VETEC.1998.686121

[16] S. Y. Le Goff, S. S. Al-Samahi, B. K. Khoo, C. C. Tsimenidis and B. S. Sharif, "Selected Mapping without Side Information for PAPR Reduction in OFDM," in IEEE Transactions on Wireless Communications, vol. 8 , no. 7, pp. 3320-3325, 2009. doi: 10.1109/TWC.2009.070463

[17] M. Al-Rayif, "Partially Orthogonal SLM in SISO OFDM System without Side Information," International Symposium on Wireless Communication Systems (ISWCS), Paris, 2012, pp. 924-928. doi: 10.1109/ISWCS.2012.6328503

[18] R. F. H. Fischer, and C. Siegl, "Performance of Peak-to-Average Power Ratio Reduction in Single- and Multi-Antenna OFDM via Directed Selected Mapping," in IEEE Transactions on Communications, vol. 57, no. 11 , pp. 3205-3208, 2009. doi: 10.1109/TCOMM.2009.11.070354

[19] Myung-Sun Baek, Mi-Jeong Kim, Young-Hwan You, and Hyoung-Kyu Song, "Semi-Blind Channel Estimation and PAR Reduction for MIMO OFDM System with Multiple Antennas," in IEEE Transactions on Broadcasting, vol. 50, no. 4, pp. 414-424, 2004. doi: 10.1109/TBC.2004.837885

[20] Yung-Lyul Lee, Young-Hwan You, Won-Gi Jeon, Jong-Ho Paik, and Hyoung-Kyu Song, "Peak-to-Average Power Ratio in MIMO-OFDM Systems using Selective Mapping," in IEEE Communications Letters, vol. 7, no. 12 , pp. 575-577, 2003. doi: 10.1109/LCOMM.2003.821329

[21] C. Ni, Y. Ma and T. Jiang, "A Novel Adaptive Tone Reservation Scheme for PAPR Reduction in Large-Scale Multi-User MIMO-OFDM Systems,' in IEEE Wireless Communications Letters, vol. 5, no. 5, pp. 480-483, 2016. doi: 10.1109/LWC.2016.258848

[22] H. Bao, J. Fang, Q. Wan, Z. Chen and T. Jiang, "An ADMM Approach for PAPR Reduction for Large-Scale MIMO-OFDM Systems," in IEEE Transactions on Vehicular Technology, vol. 67, no. 8, pp.7407-7418, 2018. doi: 10.1109/TVT.2018.2837112

[23] L. Chen, W. Chen, Y. Liu, C. Yang and Z. Feng, "An Efficient Directional Modulation Transmitter With Novel Crest Factor Reduction Technique," in IEEE Microwave and Wireless Components Letters, vol. 29, no. 8, pp. 554-556, 2019. doi: 10.1109/LMWC.2019.2923361

[24] B. M. Lee,'Energy Efficient Selected Mapping Schemes Based on Antenna Grouping for Industrial Massive MIMO-OFDM Antenna Systems,' in IEEE Transactions on Industrial Informatics, vol. 14, no. 11, pp. 48044814, 2018. doi: 10.1109/TII.2018.2803118 


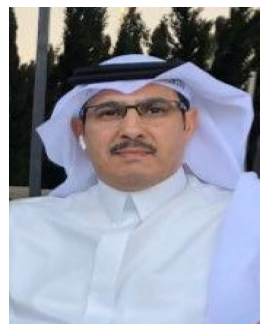

Mohammed I. Al-Rayif received his B.Sc. degree in Electrical Engineering from King Saud University, Riyadh, KSA, and his MSc and PhD degrees from Newcastle University, UK, in 2006 and 2010, respectively, in the field of communication and signal processing. From 1998-2014, he has worked in many positions in the Directorate General of Border Guard, Ministry of Interior in Saudi Arabia, such as the head of the Wire/Wireless Communication Systems (2000-2003) and the director of Communication Management (2011-2013). He was the chairman of Electrical Engineering Department and the Vice-Dean of the College of Engineering at King Khalid University, Abha, KSA (20142017). Between 2018-2019, he was a cooperative faculty member with the Department of Applied Electrical Engineering, Al-Muzahmia Branch of King Saud University. Currently, he is a full-time faculty member in the department of Applied Electrical Engineering. His research interests cover any subject under the field of communications and Signal Processing, such as MIMO/mMIMO systems, multi-Carrier systems, mm-Wave communication, and optical communications. 